\title{
Some Basic Issues of the Averaged $G$-Equation Approach to Premixed Turbulent Combustion Modeling
}

\author{
Andrei N. Lipatnikov*,a and Vladimir A. Sabel'nikov ${ }^{\mathrm{b}}$ \\ ${ }^{a}$ Department of Applied Mechanics, Chalmers University of Technology, Gothenburg, Sweden \\ ${ }^{b}$ ONERA/DEFA, Centre de Palaiseau, Chemin de la Hunière, 91761 Palaiseau, France
}

\begin{abstract}
In a recently developed and widely used premixed turbulent flame model, the so-called $G$-equation is averaged and combined with a balance equation for the variance $\overline{G^{2}} \equiv \overline{(G-\bar{G})^{2}}$, where $\bar{G}$ is an averaged $G$-field. The goal of this communication is to discuss certain basic issues relevant to the $\bar{G}$ - and $\overline{G^{\prime 2}}$-equations and, in particular, to stress that the latter equation is ill defined, because the $G$ - and $\bar{G}$-equations are physically meaningful at different surfaces. Moreover, by analyzing simple examples, it is shown that a widely accepted association of the scalar $G$ with the so-called distance function does not allow us to consider the $G$-equation to be physically meaningful in the entire flow field.
\end{abstract}

\section{INTRODUCTION}

In many premixed combustion models, the instantaneous position of a laminar flame is represented by an infinitely thin surface that (i) separates the unburned and burned gases, and (ii) moves at a speed $S_{L}$ relative to the unburned mixture. From this approach, flame propagation can be described by (i) assigning an arbitrary value $G_{0}$ to a scalar function $G$ of position vector $\vec{x}$ and time $t$ to define the flame surface as

$G(t, \vec{x})=G_{0}$,

and (ii) solving the $G$-equation $[1,2]$

$\frac{\partial G}{\partial t}+\vec{v} \cdot \nabla G=S_{L}|\nabla G|$

where $\vec{v}$ is the unburned-gas velocity vector at the surface and the function $G(t, \vec{x})$ is assumed to increase toward the burned-gas region.

Kinematic equation (2) is derived by taking the material derivative of equation (1):

$0=\frac{d G}{d t}=\frac{\partial G}{\partial t}+\left.\nabla G \cdot \frac{d \vec{x}}{d t}\right|_{G=G_{0}}$

$=\frac{\partial G}{\partial t}+\nabla G \cdot\left(\vec{v}+\vec{n} S_{L}\right)$

where $\vec{n}=-\nabla G /|\nabla G|$ is the unit normal vector to the flame surface pointing to the unburned gas.

The $G$-equation (2) has widely been used in premixed flame simulations and a number of important results

*Address correspondence to this author at the Department of Applied Mechanics, Chalmers University of Technology, Gothenburg, Sweden; Tel: +46 3177213 86; Fax: +46 311809 76; E-mail: lipatn@chalmers.se obtained by solving it have substantially contributed to premixed combustion understanding. Certainly, as any model, the $G$-equation has certain limitations discussed elsewhere [3].

Starting with the work of Kerstein, Ashurst, and Williams [4], the most common and consistent way of using the $G$-equation consists of solving it in an instantaneous laminar or turbulent velocity field. Since direct numerical simulations of turbulent flows are computationally expensive, two alternative ways of using the $G$-equation have also been proposed.

One, completely empirical approach has been used for decades (e.g. see Ref. [5]) and consists of (i) applying equation (1) to a properly chosen iso-scalar surface within the turbulent flame brush and, then, (ii) tracking the propagation of this surface by solving the following counterpart of equation (2)

$$
\frac{\partial G}{\partial t}+\overline{\vec{v}} \cdot \nabla G=S_{t}|\nabla G|
$$

where $S_{t}$ is the turbulent flame speed and $\overline{\vec{v}}$ is the Reynoldsaveraged unburned-gas velocity vector at the surface. Note that the same symbol $G$ in equations (2) and (4) designates different scalar fields, associated with the instantaneous and mean flame surfaces, respectively. Equation (4) requires a model for $S_{t}$. Moreover, to compute the mean temperature field by solving equation (4), a model for the mean flame brush thickness $\delta_{t}$ should also be invoked.

An alternative approach was put forward by Peters [6-8] and was widely used in simulations of premixed turbulent combustion within RANS [9] and LES [10,11] framework over the past years. The approach is aimed at determining not only flame surface but also flame thickness by analyzing equation (2). The governing equations of the model were formally derived as follows $[6,7]$ : 
First, the $G$-equation was "considered to be valid everywhere in the flow field" (see p. 119 in Ref. [7], i.e. velocities $\vec{v}$ and $S_{L}$ were defined not only at the flame surface but also in the entire flow field) and the scalar $G$ was split into mean and fluctuating parts, $\bar{G}$ and $G^{\prime} \equiv G-\bar{G}$, respectively. Subsequently, equation (2) was averaged to obtain the following equation for $\bar{G}(t, \vec{x})$ (see p.118 in Ref. [8])

$$
\frac{\partial \bar{G}}{\partial t}+\overline{\vec{v}} \cdot \nabla \bar{G}=S_{t}|\nabla \bar{G}|-D_{t} \bar{\kappa}|\nabla \bar{G}|,
$$

where $\kappa=\nabla \vec{n}$ is the flame curvature. In the present paper, a constant-density flow is considered to simplify discussion. Under this assumption, the Favre average used by Peters is equivalent to the Reynolds average used here and denoted by the over-bar.

Second, equation (5) was subtracted from equation (2) to obtain an equation for $G^{\prime}$.

Third, the equation (see p.118 in Ref. [8])

$$
\begin{aligned}
& \frac{\partial \overline{G^{\prime 2}}}{\partial t}+\overline{\vec{v}} \cdot \nabla \overline{G^{\prime 2}}=\nabla_{\|} \cdot\left(D_{t} \nabla_{\|} \overline{G^{\prime 2}}\right) \\
& +2 D_{t}(\nabla \bar{G})^{2}-c_{s} \frac{\bar{\varepsilon}}{\bar{k}} \overline{G^{\prime 2}}
\end{aligned}
$$

for the variance $\overline{G^{2}}(t, \vec{x})$ was obtained by averaging the $G^{\prime}$ equation multiplied by $G^{\prime}$. Here, $D_{t}$ is the turbulent diffusivity, $k$ and $\varepsilon$ are the turbulent kinetic energy and its dissipation rate, respectively, $c_{s}$ is a constant, and $\nabla_{\|}$is gradient tangential to the flame.

Finally, Peters used equations (5) and (6) as a basis for evaluating flame thickness (see p.118 in Ref. [8])

$$
\delta_{t}=\left(\frac{\overline{G^{\prime 2}}}{\nabla \bar{G} \cdot \nabla \bar{G}}\right)_{\bar{G}=G_{0}}^{1 / 2} .
$$

The goal of the present communication is to highlight certain basic issues directly relevant to the Peters approach but not yet discussed in the literature.

It should be stressed that the following discussion is only restricted to the averaged equations (5)-(7), but does not dispute the original $G$-equation which has already ranked high among combustion models.

It is worth noting also that the phrase "averaging the $G$ equation" is sometimes used to designate the evaluation of flame propagation speed by solving equation (2) in a prescribed velocity field [3]. In the present paper, this phrase means averaging the $G$-field and obtaining equations (5) and (6) for $\bar{G}$ and $\overline{G^{2}}$, as done by Peters [6-8].

\section{DISCUSSION}

The basic difficulty of averaging the $G$-field stems from the fact that the $G$-equation is physically meaningful only at the flame surface, as is clear from the above derivation, see equations (1)-(3). For instance, Peters [8] has stressed that "the quantity $G$ is a scalar, defined at the flame surface only, while the surrounding $G$-field is not uniquely defined" (see p. 92 in the cited book). However, if $G=G_{0}$ at the flame surface and "is not uniquely defined" outside it, the Reynoldsaveraged value of $G$ is equal either to $G_{0}$ if only the physically meaningful value of $G=G_{0}$ is averaged or to an arbitrary value if the entire $G$-field is processed.

Accordingly, the following two issues should be addressed in order to assess the consistency of the discussed approach with the underlying physics: First, if one invokes an extra constraint in order to uniquely define the scalar $G$ in the entire flow field, may he consider the $G$-equation to be physically meaningful outside the flame surface? Second, if this is not possible in a general case and the $G$-field is "defined at the flame surface only," are equations (5)-(7) properly substantiated from the basic viewpoint? The two issues are discussed in the next two subsections.

\section{Scalar $G$ and Distance Function}

To treat the $G$-equation "as any scalar equation in a turbulent flow field" (see p. 616 in Ref. [6]), one has first of all to uniquely define the scalar $G$ outside the flame surface. However, no rigorous scientific method has yet been elaborated to resolve this problem.

To the best of authors' knowledge, the sole method has yet been invoked for this purpose in the combustion literature. The method consists of associating the scalar $G$ with a distance function, which represents the distance between a point and the flame surface.

In certain cases (see examples 1 and 2 on pp. 98-102 [8]), the $G$-equation does model the behavior of the distance function in the entire flow field. However, this is not true in a general case. Indeed, the distance function should satisfy the following constraint [8]

$|\nabla G|=1$,

but equation (2) doesn't conserve this normalization constraint in many simple flames. A few examples follow.

First, consider a planar laminar flame stabilized at $x=0$ in a one-dimensional laminar flow with $u(x=0)=S_{L}$. If the flow velocity does not depend on $x$, the distance function $G=x$ satisfies equation (2) in the entire flow field. However, if $u$ depends on $x$, e.g. $u=S_{L^{-}} x / \tau$ and $v=y / \tau$ with $\tau$ being an arbitrary time scale, then $G=x$ does not satisfy equation (2) in the entire flow field. In a more general case of a time-dependent $\tau=\tau(t)$ (see example 3 on pp.102-104 in Ref. [8]),

$$
|\nabla G|=\exp \left(-\int_{0}^{t} \frac{d t}{\tau}\right),
$$

which is inconsistent with equation (8) at any finite $t$.

Second, consider a spherically symmetrical problem of a laminar flame that collapses in a flow of $v=v_{0}\left(r_{0} / r\right)^{2}$, provided by a point source of the unburned mixture, located at $r=0$. Equation (2) reads 


$$
\frac{\partial G}{\partial t}+v \frac{\partial G}{\partial r}=S_{L}\left|\frac{\partial G}{\partial r}\right| .
$$

If $G$ increases toward the burned-gas region and equation (8) holds, then equation (10) reduces to

$$
\frac{\partial G}{\partial t}=S_{L}-v_{0}\left(\frac{r_{0}}{r}\right)^{2},
$$

the solution to which is as follows

$$
G(t, r)-G(0, r)=\left[S_{L}-v_{0}\left(\frac{r_{0}}{r}\right)^{2}\right] t
$$

in the simplest case of a constant $S_{L}$, which is associated either with zero Markstein number $[1,8]$ or with an asymptotically large flame radius $r_{f} / \delta_{L} \rightarrow \infty$ (i.e. $v_{0} / S_{L} \rightarrow \infty$ ). However, equation (12) yields

$$
|\nabla G|=\frac{2 v_{0} t}{r}\left(\frac{r_{0}}{r}\right)^{2}+\frac{d}{d r} G(0, r),
$$

which is inconsistent with equation (8).

Furthermore, at $t \rightarrow \infty$, the flame will be stabilized at a sphere characterized by $v(r)=S_{L}$. Even if we skip equation (8), the sole stationary solution to equation (10) in the entire flow field is trivial, i.e. $G=$ const. However, this solution does not allow us to determine the flame surface. This simple case cannot be modeled by the $G$-equation (2) if it is assumed to be valid outside the flame surface.

We stress that the above examples do not mean that the use of normalization constraint (8) is in the contradiction with the $G$-equation. If the $G$-equation is considered to be physically meaningful at the flame surface only, the use of the normalization constraint is fully justified and such a method is widely utilized in numerical simulations [4,12-14]. However, in the entire flow field, the evolution of the distance function is not modeled by the $G$-equation in a general case. Hence, the use of the normalization constraint does not allow us to define the scalar $G$ outside the flame surface in a physically meaningful manner if $\vec{v}$ is considered to be the flow velocity.

\section{Averaging $G$-Equation}

Since equations (6) and (7) seem to determine the mean turbulent flame brush thickness, these equations are the key peculiarity and cornerstone of the Peters approach as compared with empirical models associated with equation (4), which invoke an independent submodel for $\delta_{t}$.

However, the disputed approach does not allow us to evaluate the thickness in a consistent manner.

First, equations (5)-(7) do not resolve the problem of evaluating $\delta_{t}$ because the three-dimensional $\nabla \bar{G}$, calculated using a solution to equation (5), is an ill-defined quantity if the equation is valid solely at a two-dimensional flame surface.
One might assume that equation (7) yields a reasonable estimate of $\delta_{t}$, because the non-uniqueness of $\nabla \bar{G}$ is compensated by the non-uniqueness of $\overline{G^{\prime 2}}$. Indeed, if $\nabla \bar{G}$ is increased by a factor $b$, then a solution $\overline{G^{2}}(t, \vec{x})$ to equation (6) is also increased by the same factor $b$, and $\delta_{t}$ calculated using equation (7) is not changed. However, this particular argument is not sufficient to make the approach consistent in a general case. To do so, one must prove that the thickness given by equation (7) is not changed when $G-G_{0}$ is multiplied by an arbitrary positive function $f(t, \vec{x})$, at least.

Moreover, even if such a proof were provided, the problem of using equation (7) would not be solved. The point is that the boundary and initial conditions to equations (5) and (6) should be specified so that the non-uniqueness of $\nabla \bar{G}$ exactly compensates the non-uniqueness of $\overline{G^{2}}$. It is unclear how we can specify such consistent conditions if $\delta_{t}$ is not known a priori.

If a solution to equation (6) is controlled by the balance between the source and sink terms on the right hand side, and does not depend on boundary and initial conditions, then

$\overline{G^{\prime 2}}=\frac{2 D_{t} \bar{k}(\nabla \bar{G})^{2}}{c_{s} \bar{\varepsilon}}$

and equation (7) yields a unique $\delta_{t}$ which is simply proportional to the turbulent length scale [8]. However, if the source and sink terms dominate, equation (6) cannot be applied to a developing flame, which is a typical mode of premixed turbulent combustion in laboratory and industrial burners [15].

Second, if both the scalar $G$ and equation (2) are physically meaningful at the instantaneous flame surface only, whereas $\bar{G}$ and equation (5) are well defined at the mean flame surface only, then any partial differential equation written in terms of $G^{\prime} \equiv G-\bar{G}$ is ill defined. Indeed, the difference $G^{\prime} \equiv G-\bar{G}$ is well defined only at points A, B, C, etc. in Fig. (1), where thin and bold curves represent instantaneous and mean flame surfaces, respectively (or on an analogous set of intersection curves in three dimensions). The evolution of a quantity so defined is hardly amenable to modeling by any conventional partial differential equation.

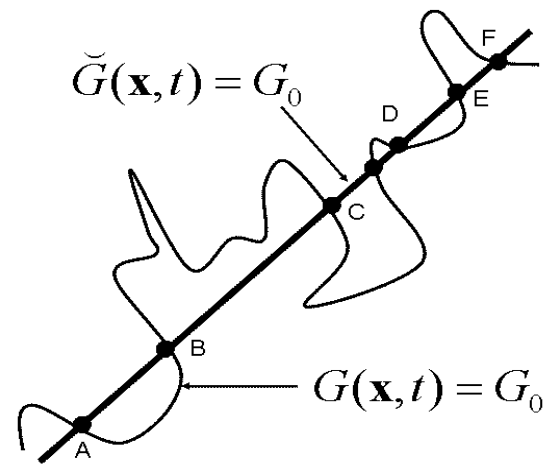

Fig. (1). Instantaneous (thin line) and mean (bold line) flame surfaces. 
Equation (6) is sometimes defended (see discussion on $\mathrm{p}$. 3058 in Ref. [16]) by referring to the paper by Oberlack, Wenzel, and Peters [17], where a $\delta_{t}$-equation (see equation 70 in the cited paper) was obtained by presenting a new scheme for averaging the $G$-field. However, the latter equation contains unclosed terms and differs substantially from equations (6) and (7). Since nobody has yet shown that the aforementioned $\delta_{t}$-equation reduces to equations (6) and (7), the two latter equations are not supported by the former.

Thus, equations (6) and (7) have not yet been substantiated properly. Let us show that equation (5) is also basically flawed.

Oberlack, Wenzel, and Peters [17] have given the following simple mathematical example, which clearly indicates that "the classical Reynolds averaging concept does not lead to a unique result for the mean $G$-field" (see p. 374 in the cited paper). If $G(t, \vec{x})$ is a solution to equation (2), then $H=\exp (G)$ also satisfies it and the same flame position may be determined either by equation (1) or by $H=\exp \left(G_{0}\right)=H_{0}$. However, if $\bar{G}(t, \vec{x})=G_{0}$ on a surface, then

$$
\bar{H}=\overline{\exp (G)} \neq \exp \left(G_{0}\right)=H_{0}
$$

on it, i.e. the mean flame surfaces are different for the two scalar fields associated with the same instantaneous surface.

To resolve the problem, Oberlack, Wenzel, and Peters [17] (i) considered the Lagrangian equations of motions of points at a flame surface, (ii) presented a new scheme for obtaining an equation for a scalar field $\breve{G}$ associated with the mean flame surface, and (iii) reported the following kinematic equation

$$
\frac{\partial \breve{G}}{\partial t}+\langle\vec{v}\rangle \cdot \nabla \breve{G}=-\nabla \breve{G} \cdot\left\langle S_{L} \vec{n}\right\rangle
$$

where $\langle q\rangle$ designates a quantity $q$ averaged by applying the scheme of Oberlack, Wenzel, and Peters [17] (rigorous definitions are given in the cited paper) and a symbol $\breve{G}$ is used instead of $G$ in order to stress that field quantities associated with the instantaneous and mean flame surfaces are different.

Equation (16) obtained by Oberlack, Wenzel, and Peters [17] differs substantially from equation (5) introduced by Peters [6-8] and the equivalence of the two equations has never been shown.

To highlight this difference, let us (i) apply equation (16) to the simplest case of a statistically stationary, planar, onedimensional flame (i.e. $\breve{G}$ depends on $x$ only, e.g. $\breve{G}=x$ with $G_{0}=0$ ) and (ii) consider the simplest case of negligible perturbations of the local structure of the instantaneous flame front by turbulent eddies (i.e. $S_{L}$ is constant). Then, in the coordinate framework linked with the flame, equation (16) reads

$$
\langle u\rangle \frac{d \breve{G}}{d x}=-S_{L}\left\langle n_{x}\right\rangle \frac{d \breve{G}}{d x} .
$$

For the constant-density problem considered, the turbulent flame speed $S_{t}$ is simply equal to the $x$-component $\bar{u}$ of the Reynolds-averaged velocity vector. If equations (5) and (16) were basically similar, then, the mean flow velocities on their left hand sides would be equal to one-another (i.e. $\bar{u}=\langle u\rangle$, note that the curvature term on the left hand side of the former equation vanishes in the considered case) and equation (17) would yield

$S_{t}=-S_{L}\left\langle n_{x}\right\rangle \leq S_{L}$

Since equation (18) is obviously wrong, equation (16) does not validate equation (5).

Furthermore, equation (16) may also be put into question. Let us (i) apply it to a planar laminar constant-density flame that moves in a quiescent mixture and (ii) impose the following initial perturbation of the flame surface

$x_{f}(0, y)=a \cos (k y)$,

where $k$ is a wave number. Since for any interval $y_{1} \leq y \leq y_{1}+2 \pi / k$, the length $\Lambda(t)$ of the instantaneous flame surface (between the planes $y=y_{1}$ and $y=y_{1}+2 \pi / k$ ) is larger than $2 \pi / k$, the mass $\rho S_{L} \Lambda$ of the burned mixture per unit time and per unit $z$-length (in three dimensions) is larger than $\rho S_{L}(2 \pi / k)$ and the flame moves at a speed

$U=S_{L} \frac{\Lambda k}{2 \pi}>S_{L}$

in the $x$-direction. Here, the laminar flame speed is again assumed to be constant.

To draw an analogy with premixed turbulent combustion, let us (i) average all quantities in the $y$-direction and (ii) consider the periodic spatial variations in $x_{f}(t, y)$ to increase the mean flame speed $U(t)>S_{L}$ and thickness similarly to turbulent eddies (to further develop the analogy, one could insert a random, uniformly distributed over period, phase $\varphi$ into the argument of the cosine). Since the averaged flame surface moves at a speed $U(t)>S_{L}$ in the $x$-direction, the surface can be determined using the following equation

$\breve{G}\left(x-\int_{0}^{t} U(\eta) d \eta\right)=G_{0}$.

Substitution of equation (21) into equation (16) yields

$U(t)=S_{L}\left\langle n_{x}\right\rangle \leq S_{L}$

in the case of a constant-density quiescent mixture. Since equations (20) and (22) contradict to one another, equation (16) cannot model this very simple case if the scalar $\breve{G}$ is associated with the mean flame surface.

Equation (16) was obtained by Oberlack, Wenzel, and Peters [17] invoking the following relation

$\frac{d\langle\vec{x}\rangle}{d t}=\langle\vec{v}\rangle+\left\langle\vec{n} S_{L}\right\rangle$, 
where $\langle\vec{x}\rangle$ is "the mean flame position" (see p.375 in Ref. [17], basically the same equation was used by Pitsch [11] for large eddy simulations). On the face of it, equation (23) appears to be very similar to the following instantaneous kinematic relation

$$
\frac{d \vec{x}_{f}}{d t}=\vec{v}+\vec{n} S_{L},
$$

used to derive the classical $G$-equation. In fact, equation (23) has been obtained using equation (24) and implicitly assuming that differentiation $d / d t$ and taking the mean $\langle\vec{x}\rangle$ commute (a similar assumption was also invoked by Pitsch [11]). However, equations (23) and (24) are substantially different.

To stress this difference, let us consider a flame stabilized in a steady laminar flow, e.g.

$$
u(y)=U-\left(S_{L}-U\right) \cos (k y) .
$$

where $\mathrm{U}>\mathrm{S}_{\mathrm{L}}$

In such a case, equation (24) results in particular in

$$
\frac{d x_{f}}{d t}=u+n_{x} S_{L},
$$

i.e. the time-derivative $d x_{f} / d t$ is positive everywhere with the exception of lines $k y=-\pi \pm 2 \pi m$. Therefore, in equation (24), $\vec{x}_{f}$ is the coordinate of a point at a flame surface, which is moved by the flow along the surface even in the stationary case.

What does the quantity $\langle\vec{x}\rangle$ in equation (23) mean? If it is "the mean flame position," then $d\langle\vec{x}\rangle / d t=0$ in the steady case studied and equation (23) with a constant $S_{L}$ results in

$$
\langle u\rangle=-S_{L}\left\langle n_{x}\right\rangle
$$

where the averaging is performed along the $y$-direction. However, equation (27) is wrong. Indeed, since $u>S_{L}$ and $-1<n_{x}$ everywhere with the exception of lines $k y=-$ $\pi \pm 2 \pi m$, any averaging method not restricted to these lines should result in a higher mean $u$ than mean $\left|S_{L} n_{x}\right|$, in contrast to equation (27).

If $\langle\vec{x}\rangle$ in equation (23) is the coordinate of a point at the mean flame surface, then $d\langle x\rangle / d t=0$ and equation (23) results in wrong equation (27) again, because the mean flame surface is parallel to the $y$-axis and, hence, a point at the surface cannot move in the $x$-direction.

Finally, if $\langle\vec{x}\rangle$ in equation (23) is the coordinate of a point at the perturbed flame surface, averaged using the scheme of Oberlack, Wenzel, and Peters [17], then $d\langle x\rangle / d t$ is positive and wrong equation (27) does not result from equation (23). However, in such a case, the quantity $\langle\vec{x}\rangle$ cannot be used to characterize the mean flame surface, which is stationary and parallel to the $y$-axis.

Thus, the relation between equations (23) and (24) is not so simple as sometimes assumed. All in all, the work by Oberlack, Wenzel, and Peters [17] does not validate the Peters approach.

\section{CONCLUSIONS}

The most critical issue for the fundamental correctness of the averaged $G$-equation approach consists of the fact that the difference $G^{\prime} \equiv G-\bar{G}$ is ill defined at any flame surface if $G$ and $\bar{G}$ are well defined at two different surfaces.

The association of the scalar $G$ with the distance function does not allow us to define $G$ in a physically meaningful manner in the entire flow field, because the evolution of the distance function is not modeled by equation (2) in many particular simple cases.

Unless the issues discussed above are resolved, the $\overline{G^{2}}$ equation (6) is ill defined and the physical relevance of results obtained using it is unclear.

\section{ACKNOWLEDGEMENT}

The first author is grateful to A. Betev and J. Chomiak for valuable discussion. The support from the Swedish Research Council (VR) is gratefully acknowledged.

\section{REFERENCES}

[1] G. H. Markstein, Nonsteady Flame Propagation. New York: MacMillan, 1964.

[2] F. A. Williams, "Turbulent combustion," in The Mathematics of Combustion, J. D. Buckmaster, Ed. Philadelphia: Society for Industrial and Applied Mathematics, 1985, pp. 97-131.

[3] P. F. Embid, A. J. Majda, and P. E. Souganidis, "Comparison of turbulent flame speeds from complete averaging and the Gequation," Phys. Fluids, vol. 7, pp. 2052-2060, August 1995.

[4] A. R. Kerstein, Wm. T. Ashurst, and F. A. Williams, "Field equation for interface propagation in an unsteady homogeneous flow field," Phys. Rev. A, vol 37, pp. 2728-2731, April 1988.

[5] S. M. Ilyashenko and A. V. Talantov, Theory and Analysis of Straight-Through-Flow Combustion Chambers. Ohio: WrightPatterson AFB, 1966.

[6] N. Peters, "A spectral closure for premixed turbulent combustion in the flamelet regime," J. Fluid Mech., vol. 242, pp. 611-629, September 1992.

[7] N. Peters, "The turbulent burning velocity for large-scale and small-scale turbulence," J. Fluid Mech., vol. 384, pp. 107-132, April 1999.

[8] N. Peters, Turbulent Combustion. Cambridge: Cambridge University Press, 2000.

[9] M. Herrmann, "Numerical simulation of turbulent Bunsen flames with a level set flamelet model," Combust. Flame, vol. 145, pp. 357-375, April 2006.

[10] H. Pitsch and L. D. de Lageneste, "Large-eddy simulation of premixed turbulent combustion using a level-set approach," Proc. Combust. Inst., vol. 29, pp. 2001-2008, 2002.

[11] H. Pitsch, "A consistent level set formulation for large-eddy simulation of premixed turbulent combustion," Combust. Flame, 143 pp. 587-598, December 2005.

[12] H. Wenzel and N. Peters, "Direct numerical simulation and modeling of kinematic restoration, dissipation and gas expansion effects of premixed flames in homogeneous turbulence," Combust. Sci. Tech., vol. 158, pp. 273-297, September 2000.

[13] H. Wenzel and N. Peters, "Scaling of production, kinematic restoration, and dissipation of the mean flame surface area," Combust. Sci. Tech., vol. 177, pp. 1095-1107, May 2005. 
[14] A. Kurenkov and M. Oberlack, "Modelling turbulent premixed combustion using the level set approach for Reynolds averaged models," Flow, Turb. Combust., vol. 74, pp. 387-407, June 2005.

[15] A. N. Lipatnikov and J. Chomiak, "Turbulent flame speed and thickness: phenomenology, evaluation, and application in multidimensional simulations," Prog. Energy Combust. Sci., vol. 28, pp. 1-73, January 2002.
[16] J. Ewald and N. Peters, "On unsteady premixed turbulent burning velocity prediction in internal combustion engines," Proc. Combust. Inst., vol. 31, pp. 3051-3058, 2007.

[17] M. Oberlack, H. Wenzel, and N. Peters, "On symmetries and averaging of the $G$-equation for premixed combustion," Combust. The ory Modell., vol. 5, pp. 363-383, July 2001.

Received: March 28, 2008

Revised: April 24, 2008

Accepted: April 24, 2008

(ㄱ. . Lipatnikov and Sabel'nikov; Licensee Bentham Open.

This is an open access article distributed under the terms of the Creative Commons Attribution License (http://creativecommons.org/license/by/2.5/), which permits unrestrictive use, distribution, and reproduction in any medium, provided the original work is properly cited. 